\title{
STRUCTURE AND THERMODYNAMICS OF LANTHANIDE AND ACTINIDE COMPLEXES IN SOLUTION
}

\author{
GREGORY R. CHOPPIN \\ Department of Chemistry \\ Florida State University, Tallahassee, Florida 32306, USA
}

\begin{abstract}
Several questions of importance in the study of lanthanide and actinide coordination compounds are reviewed. There is considerable evidence that in aqueous solution the primary coordination number is nine for the ions La(III) through $\mathrm{Nd}(\mathrm{III})$ and eight for the ions heavier than Gd(III). While it seems that some degree of covalency exists in the metal-ligand bonding a model of electrostatic bonding seems satisfactory for explaining the structure and formation of the complexes. The dehydration of the lanthanide ions upon complexation largely determines the enthalpy and entropy data. However, there seems to be a compensation effect in the hydration parts of those terms such that the free energy changes seem to reflect the metal-ligand reaction unobscured by hydration factors. A number of individual inorganic and organic ligand systems are reviewed from these viewpoints.
\end{abstract}

The lanthanide and actinide elements constitute two families of metals which often exhibit very similar chemical behaviour. This similarity is most easily observed with the cations in oxidation state III, the most common one for all the elements of the lanthanide series and for the transplutonium elements of the actinide series. This paper reviews the present state of understanding of the complexes formed in aqueous solution by the trivalent ions of these two families of elements.

The coordination chemistry of these elements has been studied neither as intensively nor as extensively as that of the transition metal ions. One reason for this is that these elements have been less available generally than the transition metal elements. Although they are fairly abundant, a lack of industrial application until a relatively few years ago caused many of the lanthanide elements to be fairly expensive. Increased interest in the use of these elements in phosphors and lasers and as catalysts as well as improvements in the methods of separation has caused a substantial reduction in their cost. The availability of the transuranium elements of the actinide series was limited to a few nuclear energy laboratories where greater emphasis was placed on research of either a more nuclear or more applied nature. This latter situation has changed as the transplutonium programme of the US Atomic Energy Commission has resulted in production of relatively large amounts of the elements as heavy as californium $(Z=98)$. However, the 
intensely radioactive nature of these elements still imposes such constraints as the availability of a good radiochemical laboratory and the use of tracer techniques for many studies.

A second major reason for the greater interest by coordination chemists in the transition metal systems is found in the rich variety of complexes formed by these metals with a multitude of ligands. By contrast, the lanthanide and actinide ions resemble the alkaline earth ions. They are type A or 'hard' acids which have a strong preference for oxygen donor ligands. In the solid it is possible to have coordination via nitrogen, sulphur etc. but in aqueous solution complexation almost always involves substitution of the solvate waters with their metal-oxygen bond by a ligand which forms another metal-oxygen bond.

Finally, much of the great interest in transition metal complexes can be attributed to the rapid and exciting evolution of the application of ligand field theory to these systems. The $f$ electrons of the lanthanide and actinide elements are rather well shielded compared to $d$ electrons. As a result, the effect of external fields is sharply reduced in complexes of the lanthanides and actinides. Moreover, the limitation of complexation in aqueous solution to oxygen donor groups places further restriction on the extent and range of possible ligand field effects. Associated with this is the absence in the lanthanide and actinide complexes of marked variations in the nature of the bonding.

Nevertheless, study of the lanthanide and actinide elements does have a number of attractive aspects. The absence of significant ligand field effects in the formation of the complexes in aqueous solutions means that other phenomena may be more easily studied. The effect of change in cation radius, through the series of 15 elements from $\mathrm{La}$ (III) to $\mathrm{Lu}$ (III), the coordination number in the two series of elements, the degree of covalency in the complexes and its variation between the two series are a few examples of the questions of interest in complex formation by these elements. Comparison of the differences between the two families may indicate the relative roles, if any, of $4 f$ and $5 f$ orbitals in the complexation. Since these trivalent cations increase the net structuring of solvent water, these elements also allow an excellent approach for studying many solution effects such as the role of solvation and solvent structure in complex formation.

First we shall discuss, in general, the questions of coordination number, ionic size and degree of covalency. Then we shall review a few specific ligand systems with emphasis on the thermodynamics of complex formation. In no way is this intended as a complete review since references have been chosen primarily for illustrative purposes.

\section{COVALENCY}

There is general but not complete agreement that the metal-ligand bonds in lanthanide complexes are predominantly electrostatic. For example, Hoard and co-workers ${ }^{1}$ have studied the structure of HLnEDTA $\cdot 4 \mathrm{H}_{2} \mathrm{O}$ in which $\operatorname{Ln}(\mathrm{III})$ has a coordination number of ten and $\mathrm{KLnEDTA} \cdot 8 \mathrm{H}_{\mathbf{2}} \mathrm{O}$ in which the coordination number of $\operatorname{Ln}(\mathrm{III})$ is $8(\mathrm{~Tb})$ or $9(\mathrm{La})$. They conclude that in these complexes the $f$-electrons of $\mathrm{Ln}^{3+}$ have no role in determining 
gross configuration or the pattern of primary bonding even though crystal field stabilization by $f$-electrons is substantial.

Techniques such as optical and n.m.r. spectroscopy have provided convincing evidence that some small degree of covalency must be present in the bonding in lanthanide complexes. Oxygen-17 chemical shifts in solution of the lanthanide perchlorates have been attributed to formation in the hydrated cations of covalent bonds involving the lanthanide $6 s$ orbital ${ }^{2}$. This interpretation has been supported by Rueben and Fiat ${ }^{3}$ in studies of proton and ${ }^{17} \mathrm{O}$ shifts in solutions of various lanthanide compounds. These results indicate that although the bonding must be primarily electrostatic, a small degree of covalent bonding between water molecules and the lanthanides is present and, moreover, remains relatively constant along the series with little effect on the lability of the water molecules of the aquo complexes.

Some additional insight into the extent of covalency has been obtained from optical spectroscopy. The small effects of ligands on the spectra of lanthanide ions is observed as peak shifts, band splitting and intensity changes. The peak shifts have been used to conclude that the metal-oxygen bond in complexes in aqueous solution is weakly covalent $t^{4}$ and involves hybrid orbitals with $4 f$ contribution. In the solid state, for example, the change in covalency from $\mathrm{Pr}-\mathrm{F}$ bonds to $\mathrm{Pr}-\mathrm{O}$ bonds has been estimated to be approximately two per cent by the same criteria of peak shifts ${ }^{5}$. A recent study of Am(III) complexes with aminocarboxylate ligands has shown larger spectral shifts in americium $f \rightarrow f$ absorption bands than for analogous lanthanide bands ${ }^{6}$. This would be consistent with less shielding of $5 f$ orbitals than $4 f$ orbitals and, possibly, a greater degree of covalency in actinide bonding than in lanthanide bonding. Certain transitions, termed hypersensitive, exhibit relatively large sensitivities to the ligands. The theory of the origin of hypersensitivity is unsettled although it is likely that both symmetry and covalency play roles ${ }^{7,8}$. Once the factors involved in hypersensitivity are understood to a satisfactory degree, the study of the effect of ligands on hypersensitive spectral transitions may be a valuable tool in lanthanide research, particularly in regard to questions of symmetry and covalency.

In summary, then, it seems reasonable to agree that the degree of covalency is small in metal-ligand bonds of the lanthanides and the structure and thermodynamics of complex formation in aqueous solution may be interpreted on the basis of electrostatic attraction. Since the $5 f$ orbitals are less shielded in the actinide elements, it is probable that the covalency is larger. However, the similarity in complexation of the two families supports the view that in the actinides, as in the lanthanides, a model of electrostatic bonding is satisfactory for interpreting complex formation.

\section{COORDINATION NUMBER}

At this time we know little about the coordination number of trivalent lanthanide and actinide ions in complexes in aqueous solution. While the coordination numbers observed in solid complexes may reflect the values for the complexes in aqueous solution, such an assumption must be approached with caution. Nevertheless, let us cite a few cases of the coordination in solids. 
The evidence for eight, nine and ten-coordination in the hydrates of the lanthanide EDTA complexes has been mentioned in the previous section. Lanthanum carbonate octahydrate, $\mathrm{La}_{2}\left(\mathrm{CO}_{3}\right)_{3} \cdot 8 \mathrm{H}_{2} \mathrm{O}$, has also been shown to be ten-coordinate ${ }^{y}$ while the monohydrate and trihydrates of the lanthanide acetylacetonates are seven and eight coordinate respectively ${ }^{10}$. The solid neodymium bromate nonahydrate, $\mathrm{Nd}\left(\mathrm{BrO}_{3}\right)_{3} \cdot 9 \mathrm{H}_{2} \mathrm{O}$, has been found to have the $\mathrm{Nd}$ (III) ion surrounded by nine water molecules ${ }^{11}$. Coordination number six has also been found in a number of systems. Thus the coordination number varies from six to ten depending upon the particular system.

Brady studied concentrated solutions of $\mathrm{ErCl}_{3}$ and $\mathrm{ErI}_{3}$ by x-ray diffraction and concluded that six water molecules are arranged octahedrally around the cation and two halide ions are located in the centre of two opposite edges of the octahedron ${ }^{12}$. Some interesting work has been reported by Karraker in which he compared the spectra of solids and solutions ${ }^{13}$. He found that the spectra of $\mathrm{Nd}(\mathrm{III})$ in dilute solutions and in crystalline $\mathrm{Nd}\left(\mathrm{BrO}_{3}\right)_{3} \cdot 9 \mathrm{H}_{2} \mathrm{O}$ were very similar. From this he concluded that the coordination number of aquo-Nd(III) is nine. As the concentration of the solutions increased in $\mathrm{HCl}, \mathrm{LiCl}$ and $\mathrm{HClO}_{4}$, the spectra also changed to resemble that of the 8 -coordinate $\mathrm{Nd}$ (III) in $\mathrm{NdCl}_{3} \cdot 8 \mathrm{H}_{2} \mathrm{O}$. The heavier lanthanides such as $\mathrm{Ho}$ (III) and $\mathrm{Er}$ (III) do not show such spectral changes so it is assumed that the coordination is unchanged for these ions as the concentration of electrolyte increases. Studies by Spedding's group on such thermodynamic properties as molal volumes, heat capacities and relative viscosities ${ }^{14}$ indicated a change in coordination number along the lanthanide series. The best interpretation of the spectral and thermodynamic data is that the hydrated ions in dilute solution have a coordination number of nine from $\mathrm{La}$ (III) to $\mathrm{Nd}$ (III) and one of eight from $\mathrm{Tb}$ (III) to $\mathrm{Lu}(\mathrm{III})$. Pm(III), Sm(III), Eu(III) and Gd(III) presumably have a mixture of these coordination numbers.

As is obvious from the variety of coordination numbers found in solid complexes, we may not assume that the same coordination numbers exist in complexes in solution as in the hydrated ions. However, studies with aminopolycarboxylate ligands have led to the suggestion that the coordination number of $\mathrm{Ln}(\mathrm{III})$ in complexes is greater than $\operatorname{six}^{15}$. Thompson provided evidence from mixed ligand complexes that coordination numbers greater than six are, in fact, more likely the norm ${ }^{16}$. He showed that six-coordinate transition metal ions would not form mixed chelates with $N$-hydroxyethylethylenediaminetriacetic acid, HEDTA, and iminodiacetic acid, IMDA. However, the lanthanides formed $\operatorname{Ln}\left(\right.$ HEDTA) (IMDA) ${ }^{2-}$ and $\operatorname{Ln}($ EDTA) (IMDA) $^{3-}$ from which Thompson concluded the coordination number to be eight for the lanthanide ions. More recently the study of other mixed complexes of the lanthanides supports a coordination number of eight ${ }^{17}$.

It seems likely, then, that in complexes the coordination number is variable and dependent on steric effects of the ligands rather than on the geometry of directed bonds. This is consistent with the electrostatic nature of the bonding. Very little has been done on coordination numbers of trivalent actinide ions in solution or in complexes and the few studies available indicate a situation very similar to that of the lanthanide ions. It has been suggested that a coordination number of ten exists for Am(III) in complexes ${ }^{6}$. 


\section{IONIC SIZE}

A favourite relationship of researchers in lanthanide complexation is that between the stability constants or some other thermodynamic parameter and the reciprocal of the cationic radius. A linear relationship would be expected from a model of purely electrostatic interaction. Invariably the ionic radii used for the lanthanides are those reported by Templeton and Dauben ${ }^{18}$ which relate to a coordination number of six, whereas, as we have seen, the actual coordination number is likely larger and may even change between $\mathrm{Nd}(\mathrm{III})$ and $\mathrm{Tb}(\mathrm{III})$; for example from nine to eight. In Figure 1 the experimental radii for $C N=6$ and calculated radii for $C N=8$ and

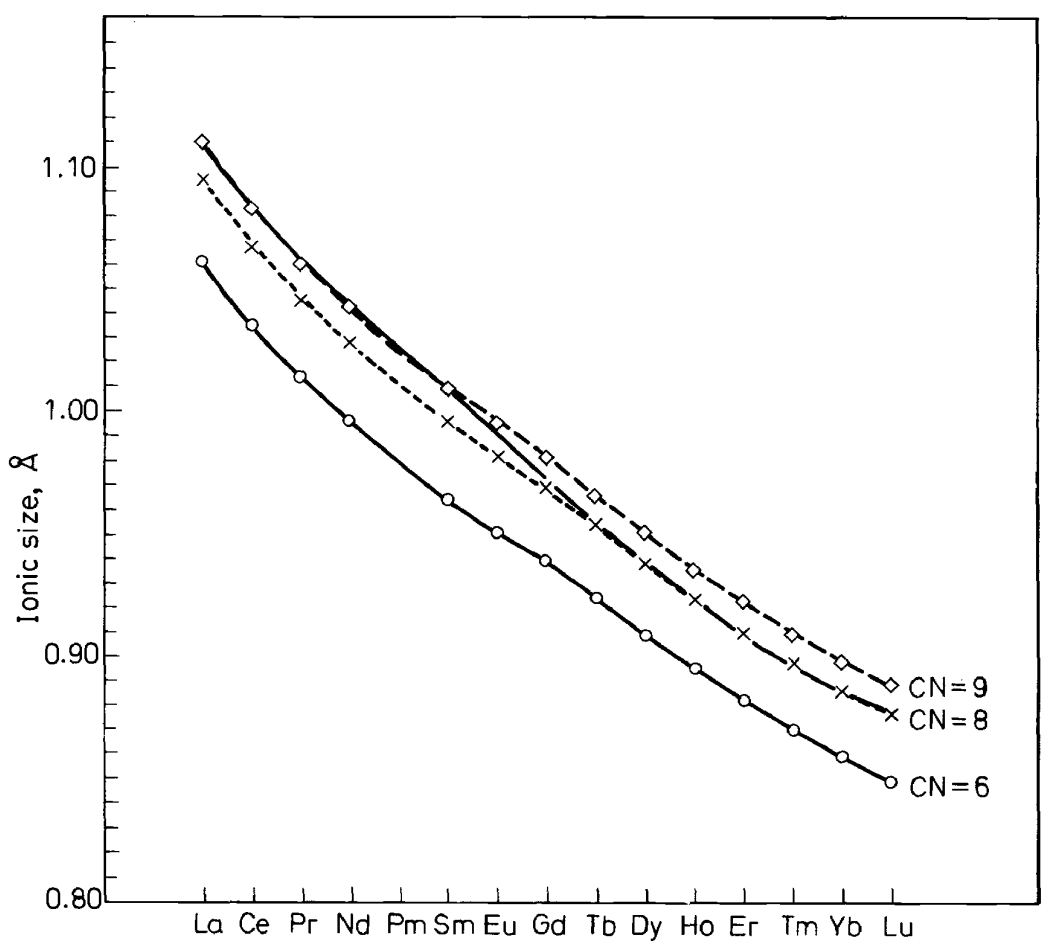

Figure 1. Ionic radii for coordination numbers six, eight and nine

$C N=9$ are presented. In the calculations a factor of 1.032 was used for $C N=8$ and 1.046 for $C N=9$ as recommended by Pauling ${ }^{19}$. The heavy line shows the trend in radii if a change from $C N=9$ to $C N=8$ occurs between $\mathrm{Nd}(\mathrm{III})$ and $\mathrm{Tb}(\mathrm{III})$. We can see that a deviation from linearity in plots involving the reciprocal of the radius may reflect use of the wrong set of values as the trend of the heavy line differs from that of the line for $C N=6$. Hopefully, sets of lanthanide ion crystal radii for $C N=8,9$ and 10 may be determined by crystallographers for use in the correlations with thermodynamic properties of these elements. However, the existence of different $\mathrm{Ln}-\mathrm{O}$ bond lengths within the same compound poses a serious question to the definition of the radius. 


\section{INORGANIC LIGANDS}

Let us now turn our attention to a consideration of some specific ligand systems. Among the inorganic ligands whose complexation has been studied with lanthanide and actinide ions are the halides, nitrates, thiocyanates and sulphates.

With the lanthanide and actinide ions, chloride, bromide and iodide form very weak complexes whose order is $\mathrm{Cl}>\mathrm{Br}>\mathrm{I}$. On the basis of the small, negative values of the enthalpy and entropy terms for the formation of the monochloro species, the complexes have been characterized as outer sphere $^{20}$. This model of solvent separated ion pairing was supported by studies of the spectra of $\mathrm{Nd}$ (III) which showed no change up to concentrations of $5 \mathrm{M}$ chloride ion ${ }^{21}$. A spectral change was observed at higher concentrations which may be related to a change in coordination number from nine to eight as proposed by Karraker.

There is no evidence for formation of inner sphere complexes of the halides with lanthanide ions. The trivalent actinides, however, are reported to form such species to the extent of about one per cent of that of the outer sphere complexation ${ }^{22}$. This corresponds to an increase of about $2.7 \mathrm{kcal}$ in the free energy as a ligand moves from an outer to an inner sphere position and a water molecule is eliminated. Even in concentrated $\mathrm{LiCl}$ solutions, only two chlorides can enter the inner sphere to form $\left[\mathrm{AmCl}_{2}\left(\mathrm{H}_{2} \mathrm{O}\right)_{x}\right]^{1+}$ with a likely coordination number of eight. The observation of the inner sphere complexation in actinides may reflect the greater probability of participation of the $5 f$ orbitals in bonding.

The nitrate complexes are slightly stronger than the halide complexes. Again, the thermodynamic data on the formation of the mononitrato species is indicative of outer sphere complexation ${ }^{23}$. The net enthalpy and entropy changes in complex formation are the result of a positive contribution to both $\Delta H$ and $\Delta S$ as a result of the disruption of the hydration layer about the cation (anion hydration is usually small) and a negative contribution to both terms from the combination of the ions in the complex. In outer sphere complexation, the hydration zone should only be slightly affected with the result being rather small values of $\Delta H$ and $\Delta S$, often negative. Ahrland has recently reviewed these arguments for a number of systems and found them generally valid ${ }^{24}$. He has pointed out that values of $\Delta H_{1}$ close to zero is a more reliable criterion of formation of outer sphere complexes since the value of $\Delta S_{1}$ depends rather strongly on the ionic strength.

Although the dominant mode of complexation is outer sphere in the nitrate complexes, both spectral ${ }^{21,25}$ and n.m.r. ${ }^{3}$ data indicate the presence of a small degree of inner sphere formation, possibly two per cent ${ }^{8}$. Moreover, it seems that the nitrate ions function as bidentate ligands in these complexes.

The thiocyanate complexes of both the actinides and lanthanides have been studied and the thermodynamic and spectral data for the monothiocyanato complexes agree with an interpretation of outer sphere formation ${ }^{21,26}$. However, in concentrated solutions of ammonium thiocyanate, elution from anion exchange resins provides evidence for the formation of such species as $\mathrm{M}(\mathrm{SCN}){ }_{4}^{1-}$ which are most likely inner sphere ${ }^{27}$. Again, the actinides seem to form these anionic species to a greater extent than do the lanthanide ions. 


\section{THERMODYNAMICS OF LANTHANIDE COMPLEXES}

As an example of complex formation with divalent inorganic ligands, let us consider the sulphate system. The stability constants for $\mathrm{LnSO}_{4}^{+}$are an order of magnitude greater than those for $\mathrm{LnNO}_{3}^{2+}$ but still rather small (ca. 20 at an ionic strength of $2.0 \mathrm{M}$ ). The enthalpy and entropy values for the lanthanide and actinide monosulphate formation are very similar and positive. From this it was concluded that an appreciable amount of inner sphere complexation was present ${ }^{28}$. Earlier, a study by infra-red spectroscopy had led to an estimate of only 12 per cent inner sphere formation ${ }^{29}$. However, spectral data ${ }^{8,30}$ as well as the rates of formation of the monosulphate complexes studied by ultrasonic absorption ${ }^{31}$ are in agreement with the interpretation from the thermodynamic data that the complexes are predominantly inner sphere in nature.

The fluoride complexes are interesting as the magnitude and the trend of the thermodynamic parameters for the formation of the monofluoride species are unusual-for monovalent inorganic ligands ${ }^{32}$. Figure 2 shows the

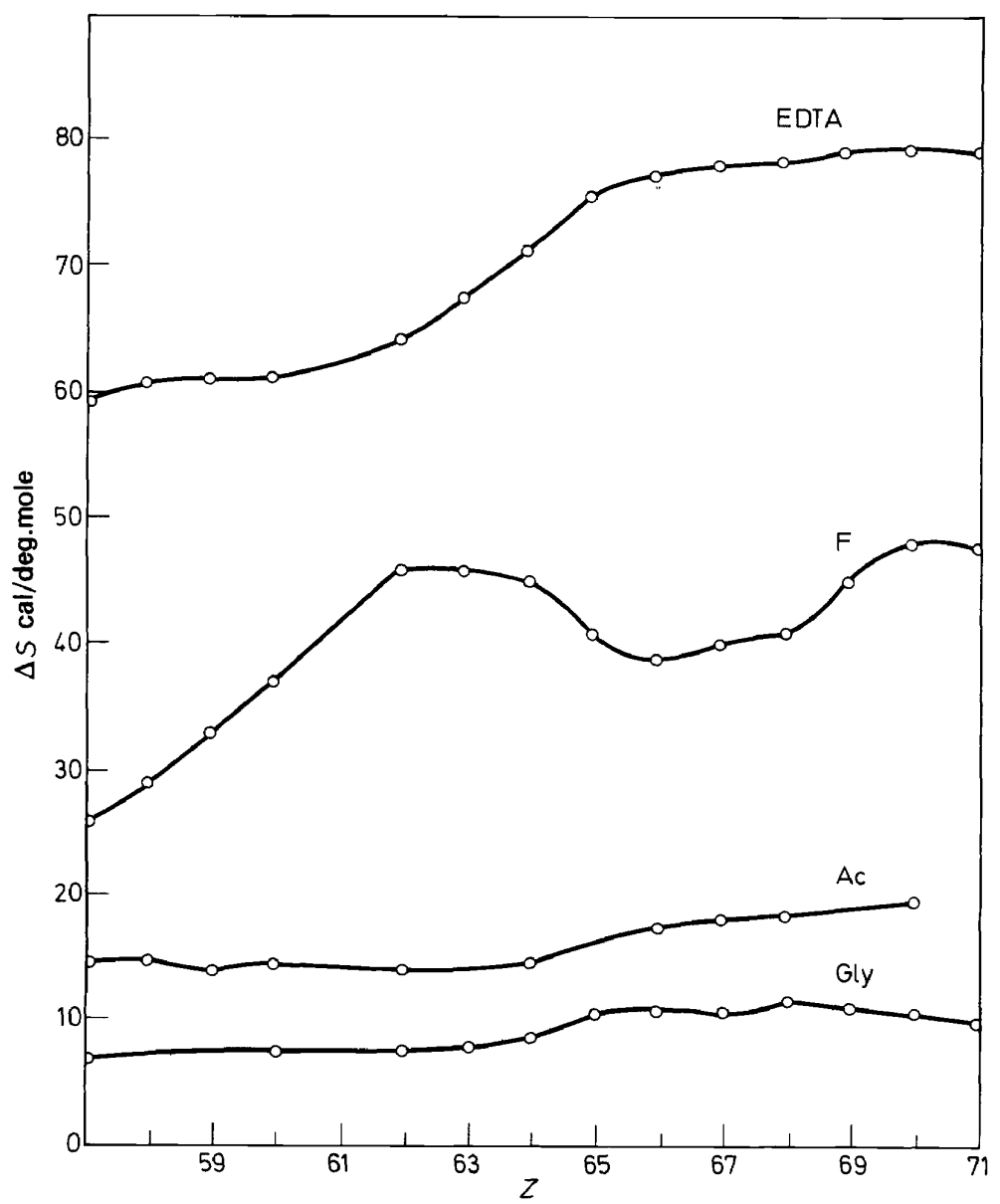

Figure 2. Entropy of formation of some monoligand complexes 


\section{GREGORY R. CHOPPIN}

entropy data compared with similar data for acetate, glycolate and the multidentate EDTA systems. The values of fluoride are surprisingly large and account for the formation of the complex since the enthalpy values are quite endothermic. These data are interpreted as evidence of inner sphere formation. Moreover, in this system we have an unusual case as the major contribution to both the entropy and the enthalpy changes comes not from the dehydration of the metal ion but rather from the disruption of the ordered hydrate

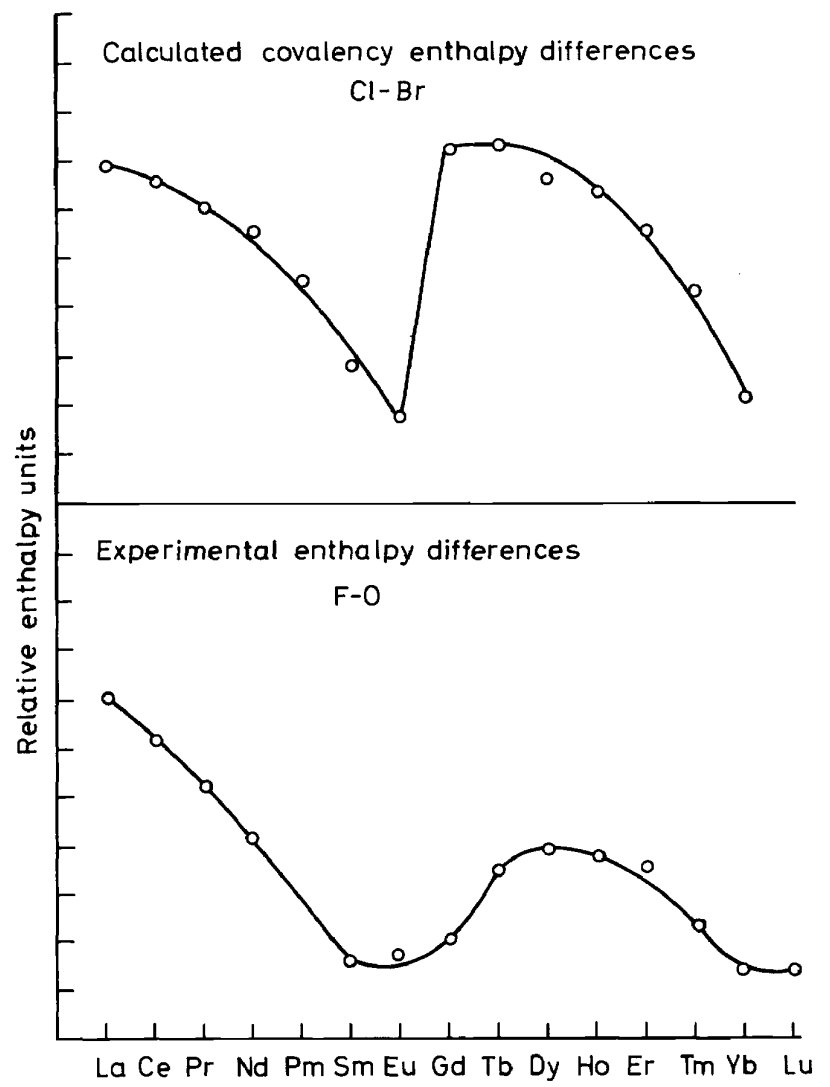

Figure 3. Enthalpy of formation of $\mathrm{LnF}^{2+}$ compared with the calculated differences in covalent bonding energy of $\mathrm{LnCl}^{2+}$ and $\mathrm{LnBr}^{2+}$

structure associated with the fluoride ion. The difference, for example, in the aqueous entropies of fluoride and acetate ions is $23 \mathrm{cal} / \mathrm{deg}$. mole which is roughly the difference between the entropy changes for formation of the monofluoride and the monoacetate complexes. The strength of the fluoride complexes is a result, then, of the entropy contribution derived from the decrease in net solvent structure when the fluoride ion is complexed rather than to any unusual metal-fluoride attraction.

The enthalpy curve for formation of the monofluoride species is also unusual (Figure 3). Since this is a rare case where a metal-oxygen bond is replaced by a metal-fluoride bond, possible covalent effects may be present. 
We have seen that in the solids, an estimate of two per cent was made for the difference in covalency between $\mathrm{Pr}-\mathrm{F}$ and $\mathrm{Pr}-\mathrm{O}$ bonds. The shape of the enthalpy curve could reflect the variation in covalent effects across the lanthanide series. To attempt to ascertain the validity of this proposal, the shape of the enthalpy curve is compared in Figure 3 with the calculated 'covalency enthalpy differences' between the lanthanide monochloride and bromide complexes based on the energy of their charge transfer bands in ethanol. The details of this calculation are to be found in ref. 32. The agreement between the two curves is not perfect but does add some support to the proposal that the enthalpy trend in the fluoride complexation reflects covalent effects. The calculated covalent curve is very similar to the trend in the energy of the $4 f^{n} 6 s^{2} \rightarrow 4 f^{n-1} 5 d^{1} 6 s^{2}$ change $^{33}$.

\section{ORGANIC LIGANDS}

Organic ligands form stronger complexes with the lanthanide and actinide ions than do inorganic ligands. The complexes of the simplest carboxylic acids such as acetate, propionate and isobutyrate are inner sphere. As in the case of the inorganic ligands, the thermodynamic data for complexation with organic ligands in aqueous solution can be interpreted as primarily reflecting the effects on the hydrate structure about the cation ${ }^{34,35}$. The formation of an inner sphere complex sufficiently disrupts the hydration about the cation to result in net entropy and enthalpy values that are positive. As a result, as in the fluoride and sulphate systems, the spontaneous formation of the complex is attributable to a favourable entropy change overcoming an unfavourable enthalpy change.

The thermodynamics of the formation of the monoacetate complexes have been compared for the lanthanide and actinides ${ }^{36}$. As in many complex systems, the stability constants (and, therefore, the free energies) of the actinides are very similar to those of the lanthanides of comparable radii. As shown in Figure 4, however, the enthalpies and entropies are found to be quite different but the difference in these terms between the actinides and lanthanides exactly balance. This 'compensation effect' can be understood by separating the thermodynamic terms into two parts - a reaction part and a hydration part :

$$
\Delta G=\Delta G^{r}+\Delta G^{h}=\Delta H^{r}-T \Delta S^{r}+\Delta H^{h}-T \Delta S^{h}
$$

If we assume that all the hydrated systems remain in equilibrium with the bulk solvent, $\Delta G^{h}=0^{37}$ and, consequently

$$
\Delta H^{h}=T \Delta S^{h}
$$

Therefore, the agreement in $\Delta G$ values for lanthanide and actinide complexing means that $\Delta G^{r}$ is similar for the two series of complexes. This is consistent with a model of ionic bonding since the cationic radii are similar. Moreover, we can conclude that the difference in $\Delta H$ and $\Delta S$ values indicates that the complexing of the actinide ions by acetate is attended by a greater dehydration than is the complexing of the lanthanide ions. The difference of 9-10 cal $/ \mathrm{deg}$. mole is consistent with the release of $0.5-1.0$ additional $\mathrm{H}_{2} \mathrm{O}$. 
GREGORY R. CHOPPIN

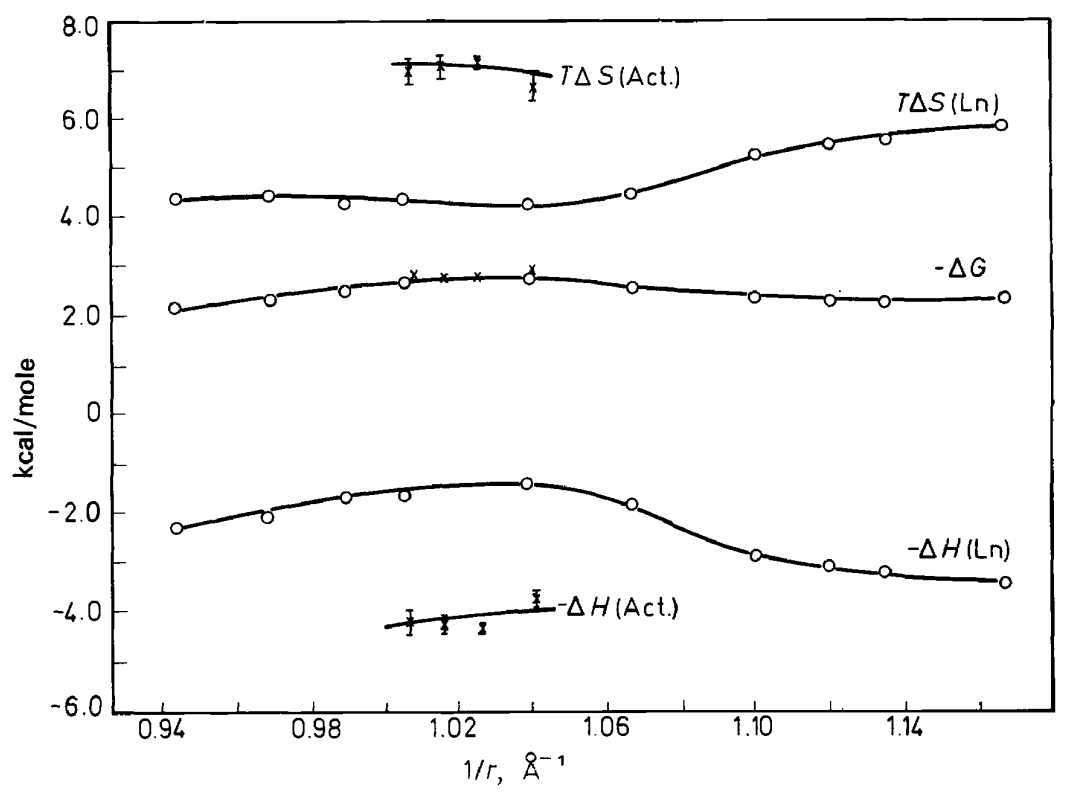

Figure 4. Thermodynamic parameters of formation of $\mathrm{MAc}^{2+}$ where $\mathrm{O}=$ lanthanides and $x=$ actinides

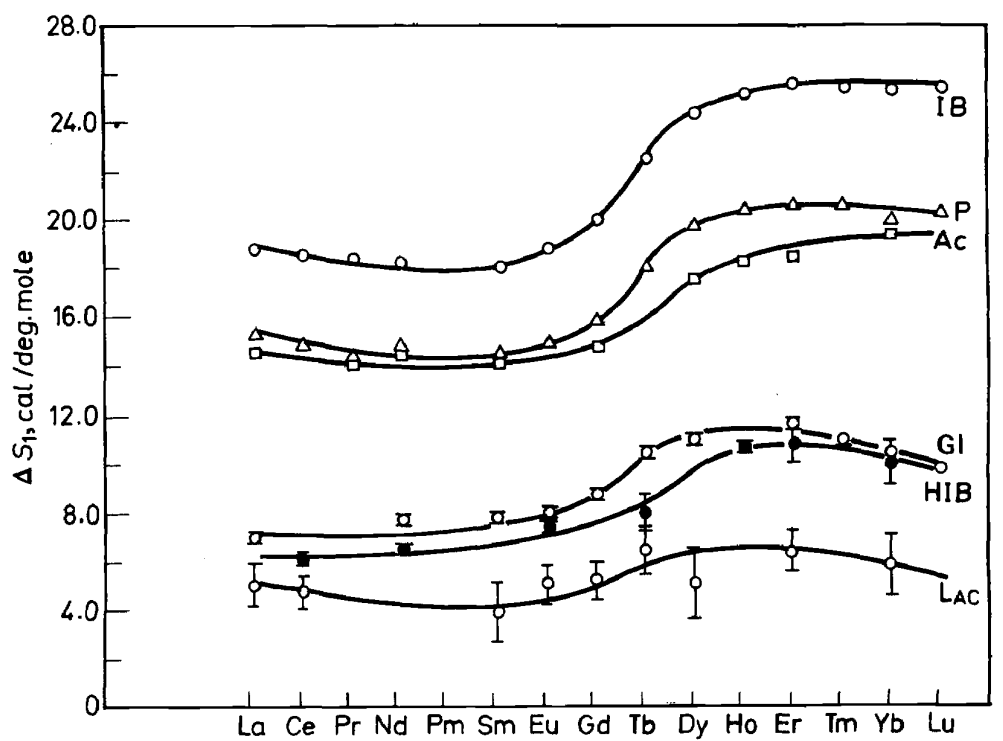

Figure 5. Entropy of formation of $\mathrm{MX}^{2+}$ complexes with carboxylate and $\alpha$-hydroxycarboxylate ligands; $\mathbf{A c}=$ acetate, $\mathbf{P}=$ propionate; $\mathbf{I B}=$ isobutyrate, $\mathrm{Gl}=$ glycolate, $\mathbf{L a c}=$ lactate, $\mathrm{HIB}=\alpha$-hydroxyisobutyrate 
The $\alpha$-hydroxycarboxylates could be expected to form bidentate chelates with these cations since they do have larger stability constants. If this does occur, it might be expected to be evidenced by a more positive entropy change. In Figure 5 the variation of the entropy for the formation of the monoligand complex is shown for three different $\alpha$-hydroxycarboxylate ligands and their simple carboxylate analogues ${ }^{38}$. Contrary to expectation, the entropy values are 6-12 cal/deg.mole smaller for chelate formation. It has been suggested that the chelate may be formed via a water molecule between the $\alpha$-hydroxy group and the cation ${ }^{39}$. This explanation has been supported by infra-red studies of the gadolinium-glycolate complex in solution ${ }^{40}$. The enthalpy values are sufficiently negative for the $\alpha$-hydroxycarboxylate complexes to overcome the lower entropy and to result in the larger stability constants compared to the values for the simple carboxylate complexes.

The formation of complexes with $\beta$-hydroxycarboxylate ligands occurs with thermodynamic changes very similar to those for the simple carboxylate analogues ${ }^{41}$. Apparently, no chelation occurs involving the $\beta$-hydroxy group even via intermediation of a water molecule. Similarly, the $\alpha$ - and $\beta$-mercaptocarboxylate ligands do not form chelates involving the mercapto group ${ }^{42}$.

A number of investigators have studied the formation of complexes with a variety of aminocarboxylate ligands. The simplest such ligand is glycine, $\alpha$-aminoacetate. The stability constants in a medium of $2.0 \mathrm{M}\left(\mathrm{NaClO}_{4}\right)$ are small and both the enthalpy and entropy for the formation of the monoglycinate species are positive for lanthanides and actinides ${ }^{43}$. We may conclude that the complex is inner sphere and is formed in these experiments (pH 3.64) by the glycinate zwitterion. By contrast, the thermodynamic values for formation of the mono- $\alpha$-picolinate complex have been interpreted as indicating chelation involving the ring nitrogen and the carboxylate group ${ }^{44}$.

In order to have comparable data obtained by a single group of investigators under identical conditions, the thermodynamic parameters for complex formation by a series of aminocarboxylate ligands have been determined $^{44,45}$. The ligands studied are shown in Figure 6. In Figures 7,8 and 9 the variations with lanthanide atomic number of the free energies, enthalpies and entropies are shown. The free energies are well behaved in the sense of increasing as expected for the probable increase in polydentate character of the complex formed. The enthalpies and entropies show no such regularities either in the order of the different ligands or in the patterns across the lanthanide series. The behaviour of the free energy values probably is the result of the hydrational compensation effects discussed earlier. If the variations in the enthalpy and entropy curves are attributable to different degrees and patterns of dehydration as well as the increasing polydentate nature of the complexation, the dehydration effects would compensate to produce free energies which would reflect the 'reaction' part of the complexation. If this supposition is valid, the free energy is the thermodynamic parameter from which information about the combination of cation and ligand is most easily obtained. In Figure 10 the free energy values for the formation of the monoligand complexes are plotted as a function of the maximum coordination sites which the ligand can occupy (acetate is included as a monodentate 
GREGORY R. CHOPPIN
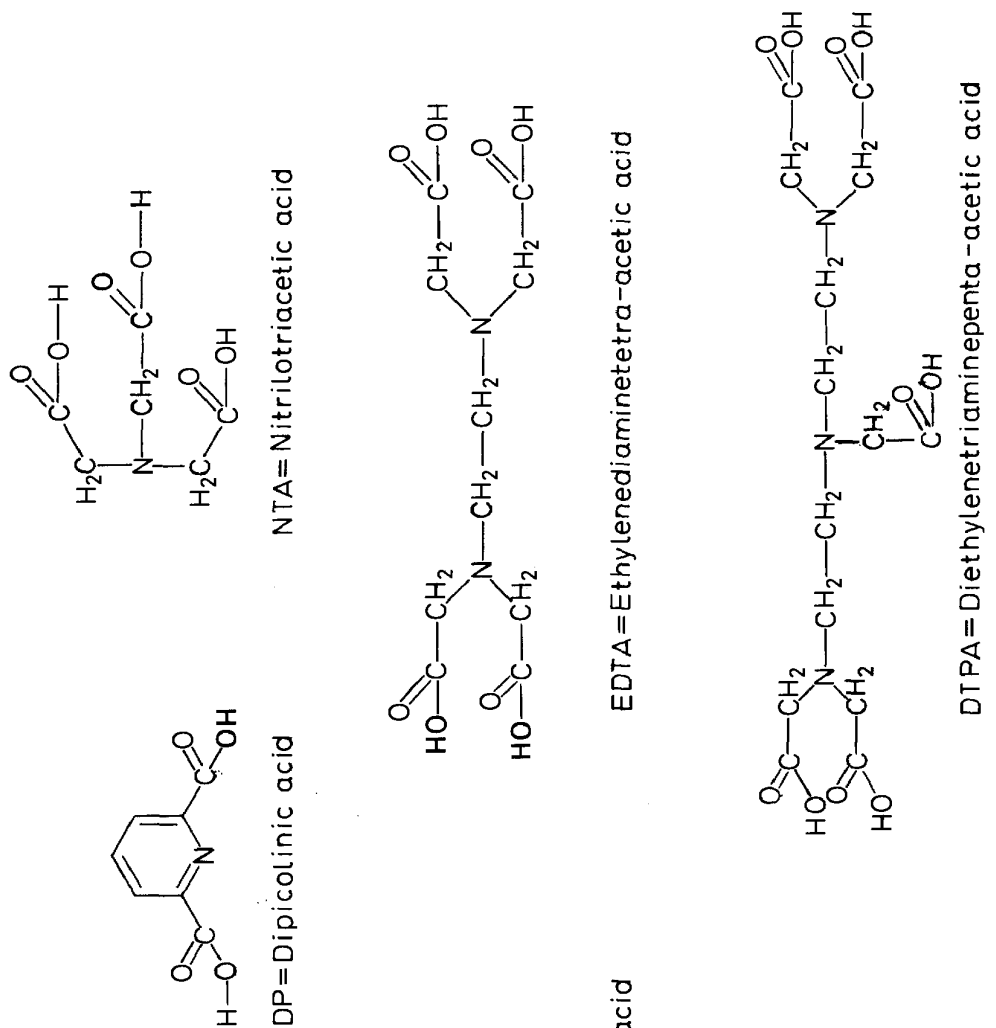

总

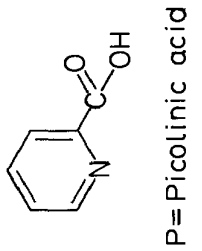

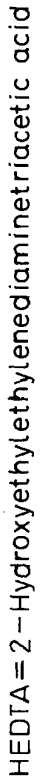

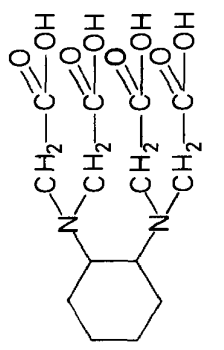




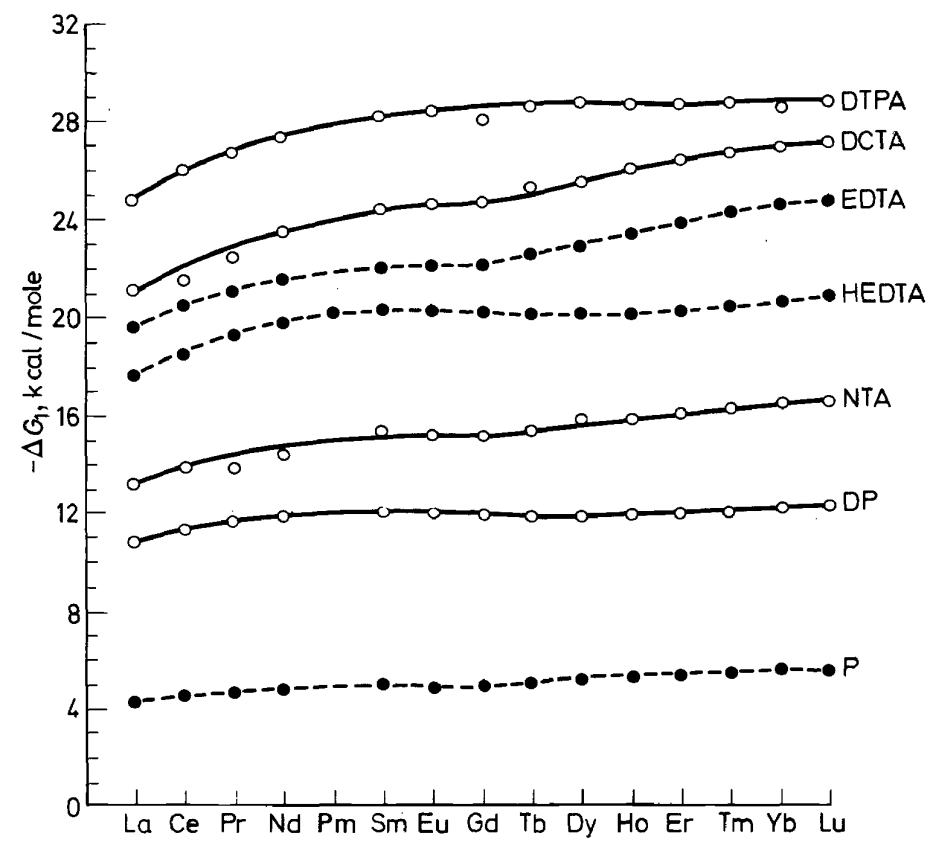

Figure 7. Free energy of formation of some monoligand complexes; $\mu=0.5 \mathrm{M}\left(\mathrm{NaClO}_{4}\right)$, $T=25^{\circ} \mathrm{C}$

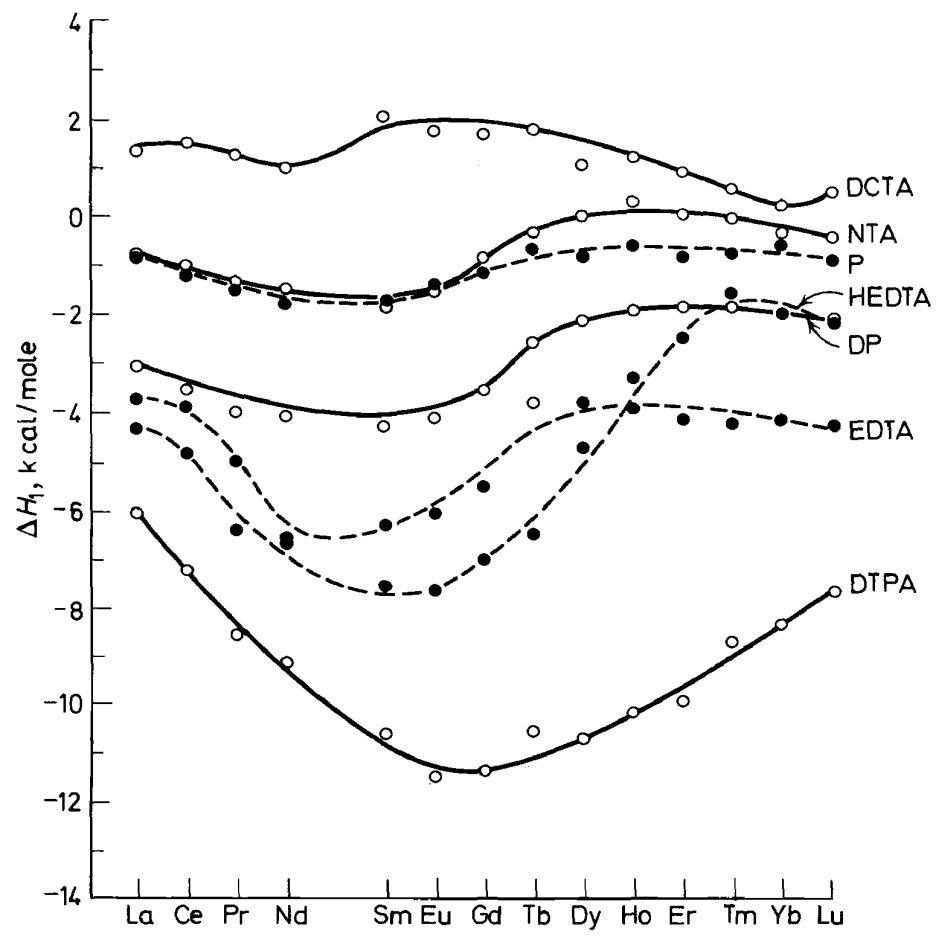

Figure 8. Enthalpy of formation of some monoligand complexes; $\mu=0.5 \mathrm{M}\left(\mathrm{NaClO}_{4}\right), T=25^{\circ} \mathrm{C}$ 


\section{GREGORY R. CHOPPIN}

ligand). The general agreement with the straight lines would suggest, but certainly not prove, that the ligands do have all the carboxylate and amino groups involved in the interaction with the metal ion. The formation of mixed complexes such as $\operatorname{Ln}\left(\right.$ EDTA) (IMDA) ${ }^{3-}$ is strong evidence that even the complexes such as LnEDTA ${ }^{1-}$ and LnDTPA $^{2-}$ still have some waters of hydration attached to the cation.

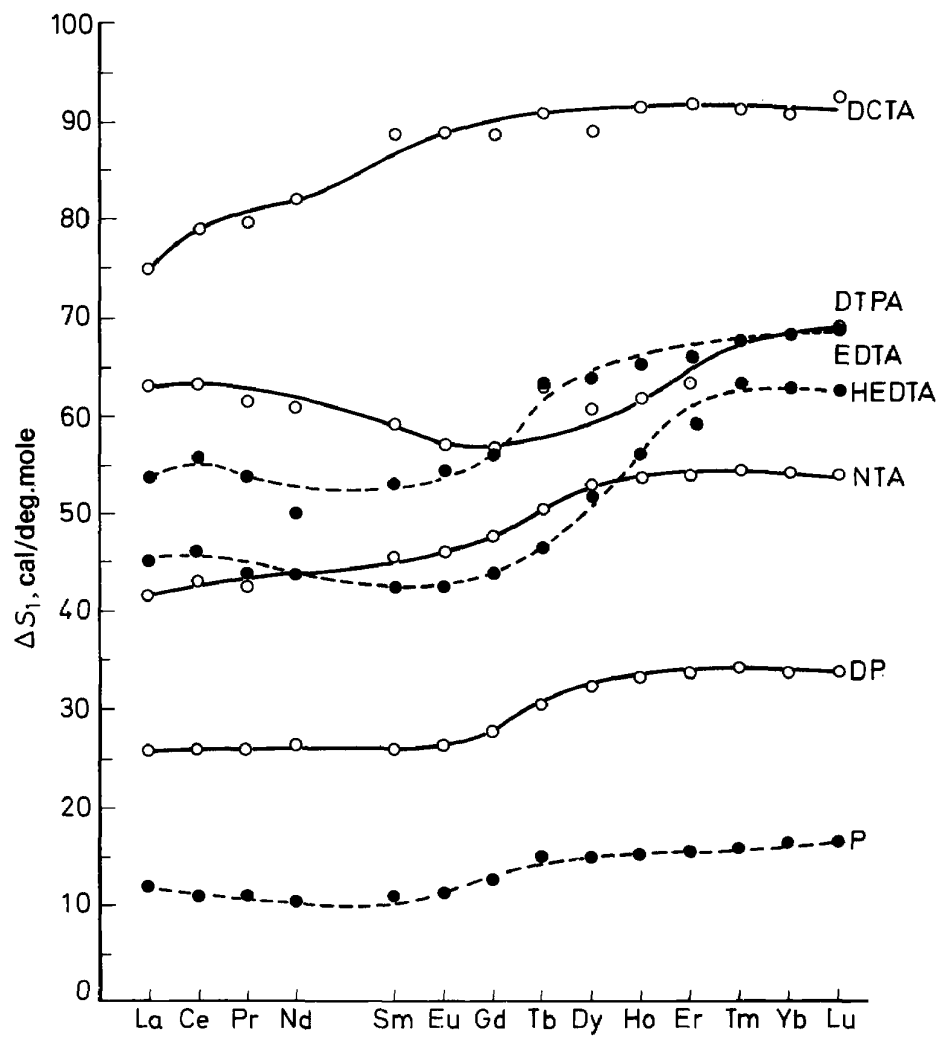

Figure 9. Entropy of formation of some monoligand complexes; $\mu=0.5 \mathrm{M}\left(\mathrm{NaClO}_{4}\right), T=25^{\circ} \mathrm{C}$

Data on the complexation of the trivalent actinides are relatively scarce. The few stability constants measured are in relatively close agreement with the analogous lanthanide data although the actinide complexes are, apparently, a little stronger ${ }^{46}$. As we learned with the acetate complexing, this is no basis for a priori assumptions that similar agreement exists for the enthalpy and entropy changes. Unfortunately, it is very difficult to perform calorimetric experiments on the actinides and the temperature coefficient technique for enthalpy is not always sufficiently reliable. 


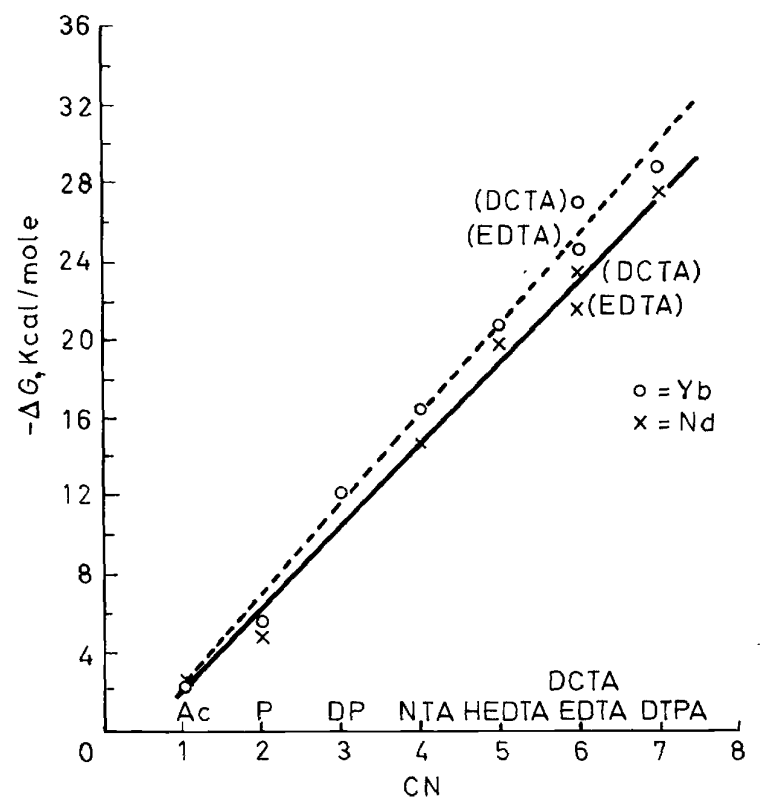

Figure 10. Relationship between the free energy of formation of MX and the maximum donor sites of the ligand

\section{HYDRATION THERMODYNAMICS}

The entropy and enthalpy data for complex formation shown in the various figures are not linear with atomic number or ionic radius. Based on the curves of the entropies for the carboxylate and $\alpha$-hydroxycarboxylate systems (Figure 5), it was proposed that two different hydration numbers exist in the lanthanide series ${ }^{35}$. The ions from $\mathrm{La}$ to $\mathrm{Nd}$ have a similar size hydration layer while the ions from Dy to Lu have a larger one. The intermediate ions $\mathrm{Pm}$ to $\mathrm{Tb}$ represent a transitional group. The data of Spedding's group ${ }^{14}$ are in agreement with this model and, in fact, it is tentatively mentioned in their papers as a possibility. We used conductance data ${ }^{47}$ to estimate the hydration numbers to be 13 for the $\mathbf{L a}$ to $\mathrm{Nd}$ ions and 14 for the heavier Dy to Lu group. Little reliance can be placed on the calculated values but it would seem proper to assume that the hydration number is approximately ten per cent larger for the heavier ion group. This is not to be confused with the primary coordination number for which the likely values are nine for the lighter La-Nd group and eight for the heavier group. Padova has used molar volume data to calculate hydration numbers of approximately nine for $\mathrm{La}$ to $\mathrm{Nd}$ and 11 for $\mathrm{Dy}$ to $\mathrm{Lu}^{18}$.

Such a difference in hydration number should be reflected in the entropies of hydration of the lanthanide ions. From measurements of the solubilities and heats of solution of the lanthanide iodates, our group was able to calculate a set of hydration entropies which did, indeed, indicate the existence of different hydration along the lanthanide $\operatorname{series}^{49}$. Recently, calculations 
based on the enthalpies and free energies of solution of the hydrated chlorides were believed to show no evidence for the change in hydration number ${ }^{50}$. I have renormalized our data to the new value for gadolinium reported in ref. 50 and the two sets of data are shown in Figure 11. The values are $\Delta S_{h}$, the entropy change for the hydration of the gaseous ion. The data are only in significant disagreement for the lighter lanthanides. Both sets of values, however, show the trends expected if there is a difference in hydration between the lighter and heavier lanthanides. Cobble saw no evidence for this as he considered only $S_{a q}^{0}$, the partial molal ionic entropy in solution, rather than $\Delta S_{h}$, the entropy of hydration. It is the latter term which would more clearly show hydration effects. The difference in $\Delta S_{h}$ between the lighter and heavier groups is roughly $10-20$ per cent, in agreement with the differences in estimated hydration numbers.

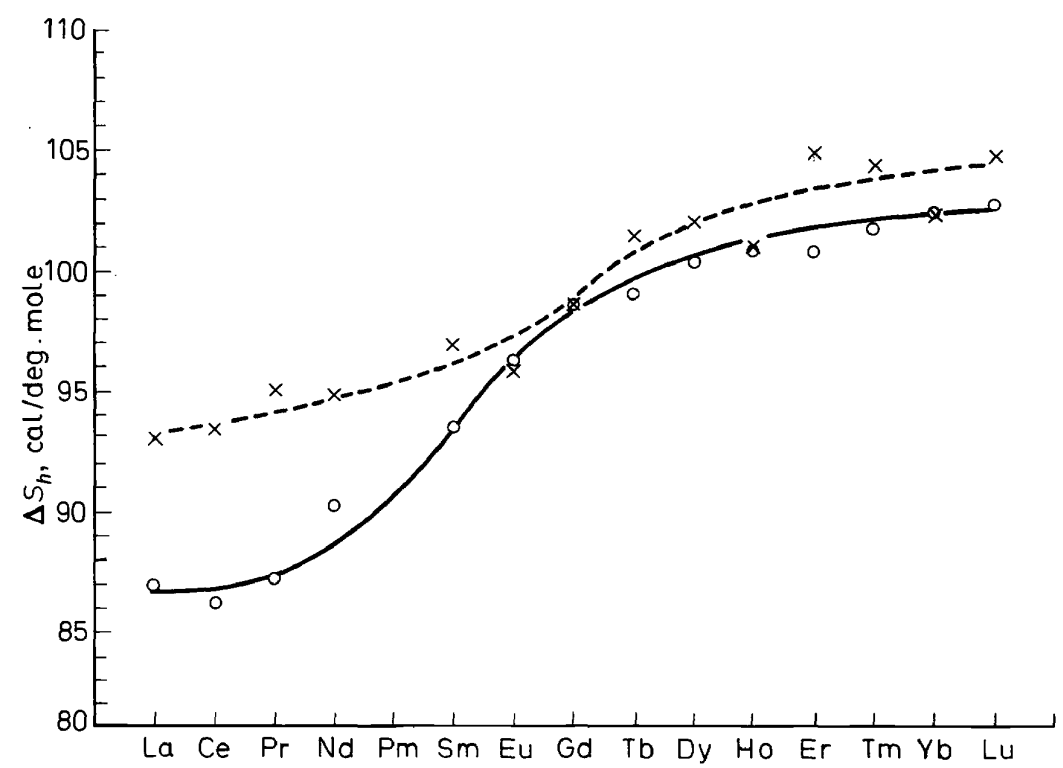

Figure 11. Variation of $\Delta S_{h}$ with atomic number in the lanthanide series: $0=$ Bertha and Choppin, ref. $49, \times=$ Hinchley and Cobble, ref. 50

The $\Delta S_{h}$ data can be used in a rough test of the idea that the thermodynamics of lanthanide complexing in solution can be attributed primarily to cation dehydration. Let us consider the complexation with EDTA. Defining $\Delta S_{g}$ as the entropy of complexation in the gas phase and $\Delta S_{s}$ as that in aqueous solution we can write:

$$
\Delta S_{g}=\Delta S_{s}-\Delta S_{h}\left(\operatorname{LnEDTA}^{1-}\right)+\Delta S_{h}\left(\mathrm{Ln}^{3+}\right)+\Delta S_{h}\left(\mathrm{EDTA}^{4-}\right)
$$

$\Delta S_{h}\left(\right.$ EDTA $\left.^{4}\right)$ is constant and we assume $\Delta S_{h}\left(\right.$ LnEDTA $\left.^{1-}\right)$ is small and relatively constant, so the equation can be rearranged:

$$
\Delta S_{g}^{\prime}=\Delta S_{g}-\Delta S_{h}\left(\mathrm{EDTA}^{4-}\right)+\Delta S_{h}\left(\operatorname{LnEDTA}^{1-}\right)=\Delta S_{s}+\Delta S_{h}\left(\operatorname{Ln}^{3+}\right)
$$


In Figure 12 the experimental values for $\Delta S_{s}$ and $\Delta S_{h}\left(\operatorname{Ln}^{3+}\right)$ and their sum, $\Delta S_{g}^{\prime}$, are plotted. It is readily apparent that addition of $\Delta S_{h}\left(\mathrm{Ln}^{3+}\right)$ has corrected most of the variation in $\Delta S_{s}$ which is to be expected if the dehydration which accompanies complex formation is the primary factor in establishing the shape of the curve of the entropies of complexation.

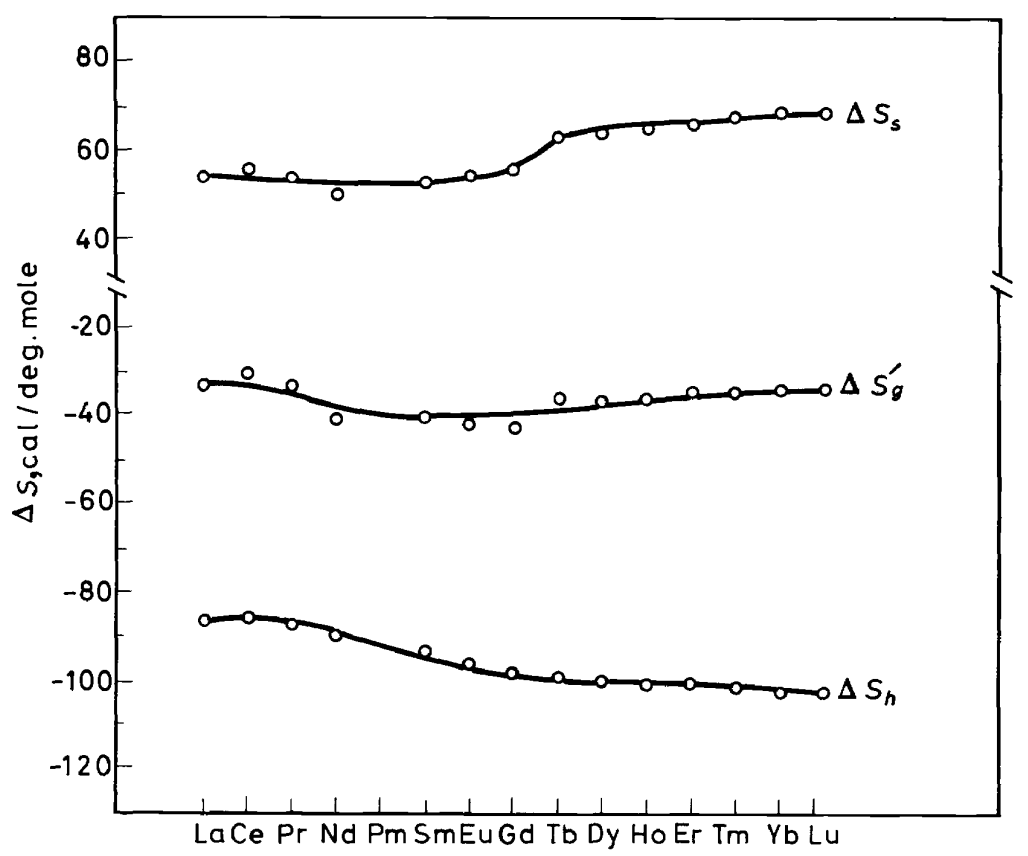

Figure 12. Effect of correction of entropy of complex formation of LnEDTA ${ }^{1-}, \Delta S_{s}$, by the entropy of hydration, $\Delta S_{h}$

\section{SUMMARY}

The trivalent lanthanide and actinide ions form predominantly outer sphere complexes with the halides (except fluoride), thiocyanate and nitrate anions. The sulphate complexes are a mixture of outer and inner sphere. The organic ligands form inner sphere complexes. The thermodynamic parameters of formation of the complexes are determined by the dehydration of the metal cation and the combination of the cation and anion. For outer sphere complexes, the enthalpy and entropy changes are usually both small, often negative. However, typically inner sphere complexation is the result of a favourable entropy change predominating over an unfavourable enthalpy change.

The coordination numbers of these cations are large, probably 8 to 10 , with evidence for a change (e.g. from 9 to 8 ) in the lanthanide series. The bonding may be interpreted on the basis of an ionic model. As a result, it is better to view the structure of the complexes not in terms of specific geometries 
induced by hybrid orbitals but as the optimal packing allowed by the cationligand radius ratios.

\section{ACKNOWLEDGEMENT}

The author wishes to acknowledge the support of the Atomic Energy Commission by AEC Contract AT(40-1)-1797.

\section{REFERENCES}

${ }^{1}$ M. D. Lind, B. Lee and J. L. Hoard, J. Am. Chem. Soc. 87, 1611 (1965); 87, 1612 (1965).

${ }^{2}$ W. B. Lewis, J. A. Jackson, J. F. Lemons and H. Taube, J. Chem. Phys. 36, 694 (1962).

${ }^{3}$ J. Rueben and D. Fiat, J. Chem. Phys. 51, 4909 (1969); 51, 4918 (1969)

${ }^{4}$ L. I. Katzin and M. L. Barnett, J. Phys. Chem. 68, 3779 (1964).

${ }^{5}$ E. Y. Wong, O. M. Stafsudd and D. R. Johnston, J. Chem. Phys. 39, 786 (1963).

${ }^{6}$ A. D. Site and R. D. Baybarz, J. Inorg. Nucl. Chem. 31, 2201 (1969).

${ }^{7}$ D. E. Henrie and G. R. Choppin, J. Chem. Phys. 49, 477 (1968).

${ }^{8}$ K. Bukietynska and G. R. Choppin, J. Chem. Phys. 52, 2875 (1970).

${ }^{9}$ D. B. Shinn and H. A. Eick, Inorg. Chem. 7, 1340 (1968).

10 J. A. Cunningham, D. E. Sands and W. F. Wagner, Inorg. Chem. 6, 499 (1967);

M. F. Richardson, Inorg. Chem. 8, 22 (1969).

${ }^{11}$ L. Helmholz, J. Am. Chem. Soc. 61, 1544 (1939).

${ }^{12}$ G. W. Brady, J. Chem. Phys. 33, 1079 (1960).

${ }^{13}$ D. G. Karraker, Inorg. Chem. 7, 473 (1968).

${ }^{14}$ F. H. Spedding, M. J. Pikal and B. O. Ayers, J. Phys. Chem. 70, 2440 (1966);

K. C. Jones, J. Phys. Chem. 70, 2450 (1966);

M. J. Pikal, J. Phys. Chem. 70, 2430 (1966).

${ }^{15}$ G. Anderegg, P. Nageli, F. Muller and G. Schwarzenbach, Helv. Chim. Acta, 42, 827 (1959).

${ }^{16}$ L. C. Thompson and J. A. Loraas, Inorg. Chem. 2, 89 (1963).

${ }^{17}$ N. A. Kostromina, K. B. Yatsimirskii, T. V. Ternovaya and N. N. Tananaeva, Proc. 3rd Symp. Coor. Chem. (Debrecen), Akademiai Kiado: Budapest (1970).

${ }^{18}$ D. H. Templeton and C. H. Dauben, J. Am. Chem. Soc. 76, 5237 (1954).

${ }^{19}$ L. Pauling, The Nature of the Chemical Bond, 3rd Edition, Cornell University Press: Ithaca, N.Y. (1960).

${ }^{20}$ G. R. Choppin and P. J. Unrein, J. Inorg. Nucl. Chem. 25, 387 (1963).

21 G. R. Choppin, D. E. Henrie and K. Buijs, Inorg. Chem. 5, 1743 (1966).

${ }^{22}$ M. Shiloh and Y. Marcus, J. Inorg. Nucl. Chem. 28, 2725 (1966);

M. Givon, J. Inorg. Nucl. Chem. 31, 1807 (1969);

M. Givon, Israel J. Chem., 7, 31 (1969).

${ }^{23}$ G. R. Choppin and W. F. Strazik, Inorg. Chem. 4, 1250 (1965).

${ }^{24}$ S. Ahrland, Proc. 3rd Symp. Coor. Chem. (Debrecen), Akademiai Kiado: Budapest (1970).

${ }^{25}$ I. Abrahamer and Y. Marcus; Inorg. Chem. 6, 2103 (1967).

${ }^{26}$ G. R. Choppin and J. Ketels, J. Inorg. Nucl. Chem. 27, 1335 (1965).

27 J. P. Surls and G. R. Choppin, J. Inorg. Nucl. Chem. 4, 62 (1957).

${ }^{28}$ R. G. de Carvalho and G. R. Choppin, J. Inorg. Nucl. Chem. 29, 725, 737 (1967).

${ }^{29}$ R. Larsson, Acta Chem. Scand. 18, 1923 (1964).

30 J. C. Barnes, J. Chem. Soc. 3880 (1964).

${ }^{31}$ D. P. Fay, D. Litchinsky and N. Purdie, J. Phys. Chem. 73, 544 (1969).

32 J. B. Walker and G. R. Choppin, Lanthanide/Actinide Chemistry, Advances in Chemistry Series No. 71, American Chemical Society, Washington, D.C. (1967).

${ }^{33}$ M. Fred, Lanthanide/Actinide Chemistry, Advances in Chemistry Series No. 71. American Society, Washington, D.C.,(1967).

${ }^{34}$ I. Grenthe, Acta Chem. Scand. 18, 293 (1964).

${ }^{35}$ G. R. Choppin and A. J. Graffeo, Inorg. Chem. 4, 1254 (1965).

${ }^{36}$ G. R. Choppin and J. K. Schneider, J. Inorg. Nucl. Chem. 32, 3283 (1970).

37 D. J. G. Ives and P. D. Marsden, J. Chem. Soc. 649 (1965).

${ }^{38}$ G. R. Choppin and H. G. Friedman Jr, Inorg. Chem. 5, 1599 (1966). 


\section{THERMODYNAMICS OF LANTHANIDE COMPLEXES}

39 I. Grenthe, Acta Chem. Scand. 18, 283 (1964).

40 R. Larsson, Acta Chem. Scand. 19, 783 (1965).

41 A. D. Jones and G. R. Choppin, J. Inorg. Nucl.Chem. 31, 3523 (1969).

42 G. R. Choppin and L. A. Martinez-Perez, Inorg. Chem. 7, 2657 (1968).

43 S. P. Tanner and G. R. Choppin, Inorg. Chem. 7, 2046 (1968).

44 T. F. Gritmon. 'Thermodynamics of the lanthanides'. Ph.D. Dissertation, Florida State University, Tallahassee (1968).

45 M. P. Goedken, 'Polyaminocarboxylate complexes of the lanthanides', Thesis, Florida State University, Tallahassee (1969).

46 A. D. Jones and G. R. Choppin, Actinide Rev. 1, 311 (1969).

${ }^{47}$ F. H. Spedding, P. E. Porter and J. M. Wright, J. Am. Chem. Soc. 74, 2055 (1952).

48 J. Padova, J. Phys. Chem. 71, 2347 (1967).

49 S. L. Bertha and G. R. Choppin, Inorg. Chem. 8, 613 (1969).

${ }^{50}$ R. J. Hinchey and J. W. Cobble, Inorg. Chem. 9, 917 (1970). 